\title{
Tratamiento de la maloclusión clase II-1 con mini-implantes: reporte de caso
}

Treatment of malocclusion class II-1 with mini-implants: a case report

Aylin Gallegos-Salazar ${ }^{1,2,3, a}$, Jose Vidalón-Castilla ${ }^{2,3, b}$

\section{RESUMEN}

Se han diseñado diferentes dispositivos, para optimizar el anclaje, el cual es esencial en el tratamiento de las maloclusiones, como la corrección de la maloclusión clase II división 1. Se presenta el caso de una paciente de 22 años de edad, dolicocéfalo, dolicofacial, perfil convexo, con maloclusión clase II-1 por protrusión maxilar. Se realiza máxima retracción anterosuperior con mini-implantes usando un vector de fuerza intermedio. Este se utiliza cuando se desea poca o ninguna alteración del plano oclusal con un movimiento a cuerpo entero de los dientes anterosuperiores. La longitud del gancho de retracción fue de $8 \mathrm{~mm}$ y la inserción del mini implante fue a $8 \mathrm{~mm}$ también desde la unión cemento adamantina (UCA), para que el vector de fuerza pase por el centro de rotación del segmento anterior (ubicado entre el incisivo lateral y canino, a $7 \mathrm{~mm}$ aproximadamente sobre el área cervical).

PALABRAS CLAVE: Ortodoncia, retracción, anclaje. (DeCS, Bireme) 


\section{SUMMARY}

Over time different devices like the mini-implants have been designed to optimize the anchorage. The anchorage is essential for orthodontic treatment, as the correction of malocclusion Class II division 1. We present a 22 years old patient, midbrain, dolicofacial, convex, malocclusion Class II-1 by maxillary protrusion, maximum anterior retraction with mini-implants was performed using a medium pull vector, this is used when little or no alteration of the occlusal plane with a whole body motion of the upper front teeth is needed. The length of the hook was $8 \mathrm{~mm}$ and the insertion of the mini implant was $8 \mathrm{~mm}$ from the union cement enamel (UCA), so that the force vector passes through the center of rotation of the anterior segment (located between the lateral incisor and canine to approximately $7 \mathrm{~mm}$ on the cervical area).

\section{KEYWORDS: Orthodontic, retraction, anchorage. (MeSH, NLM)}

\section{INTRODUCCIÓN}

La maloclusión clase II puede ser dental y/o esquelética, se caracteriza por un prognatismo maxilar, retrognatismo mandibular o una combinación de ambos, con variaciones de tipo oclusal y neuromuscular (1).

Dentro de las posibilidades actuales del tratamiento de la maloclusión clase II-1 por protrusión maxilar, se encuentra la máxima retracción con mini-implantes. La fase de retracción anterior representa una importante etapa en el tratamiento ortodóntico, en la cual el ortodoncista necesita mantener o alcanzar ciertos objetivos como son: la relación canina, molar, corrección del Over Jet (OJ) y Over Bite (OB), coincidencia de la línea media, además de mejorar el perfil facial, para que estos objetivos sean alcanzados se necesita de una óptima administración de las unidades de anclaje (1).

Actualmente, se puede lograr un anclaje esquelético, con el uso de los mini-implantes, los cuales han mostrado ser eficaces como método de control del anclaje. Éstos tienen muchos beneficios, tales como: facilidad de colocación y eliminación, costo mínimo, se pueden colocar en el hueso alveolar sin dañar a las raíces de los dientes debido a su tamaño, aplicación de fuerza ortodóntica de inmediato, el tiempo de tratamiento se reduce de manera efectiva, se pueden realizar diversos movimientos dentales y no requieren de la colaboración del paciente (2).

El primer artículo publicado sobre el anclaje ortodóncico mediante sistemas de implantes fue presentado por Gainsforth y Higley en 1945, insertaron tornillos de vitallium en la rama mandibular de 5 perros y aplicaron elásticos que se extendían desde el tornillo al gancho de un aparato fijado en el maxilar, con el objetivo de distalización. Todos los tornillos se aflojaron no pudiendo mantener una fuerza efectiva luego de un lapso de 16 a 31 días (3).

Brånemark (Suecia 1970), descubrió de manera accidental la oseointegración, la cual consiste en una conexión íntima, directa, funcional y mantenida en el tiempo, entre el hueso y un implante de titanio sometido o no, a carga (3).

En 1983 Creekmore y Eklund, colocaron tornillos de vitallium de pequeño tamaño debajo de la espina nasal anterior. Diez días después de su colocación comenzaron a ejercer fuerzas intrusivas con hilos elásticos lográndose una exitosa intrusión de $6 \mathrm{~mm}$ de los incisos centrales superiores, sin movilidad del tornillo durante la aplicación de la fuerza (3).

El material de fabricación de los mini-implantes es el titanio grado $\mathrm{V}$ y se utiliza con una superficie pulida en lugar de la micro-arenada de los implantes protésicos, ya que no se busca la osteointegración (3). Actualmente, podemos encontrar mini-implantes con dimensiones de $4 \mathrm{~mm}$ a $12 \mathrm{~mm}$ de longitud y de $1,2 \mathrm{~mm}$ a $2 \mathrm{~mm}$ de diámetro. La selección de las dimensiones dependerá de la zona en la que se coloque el mini-implante (3).

\section{Indicaciones para realizar una máxima retracción con mini-implantes}

El uso de mini-implantes en la fase de cierre de espacios beneficiará principalmente a pacientes con: $(4,5)$

1. Dificultades para colaborar con el uso de aparatos 
extraorales, elásticos u otros métodos de anclaje.

2. Necesidad de máximo anclaje en el maxilar superior $y / 0$ inferior.

3. Unidad de anclaje comprometida, es decir número reducido de dientes por reabsorción o secuelas de enfermedad periodontal.

4. Plano oclusal inclinado o no, en la región anterior.

Contraindicaciones para realizar una máxima retracción con mini-implantes

El uso de mini-implantes permite grandes retracciones del sector anterior, pero se debe tener en consideración diversos factores, estos son: $(4,5)$

1. Una gran retracción de incisivos puede causar disminución de la estética facial especialmente en pacientes con retrusión mandibular.

2. En el caso de pacientes que presentan proyecciones labiales ligeramente pronunciadas pero estéticamente agradables, una disminución significativa en la proyección de los labios puede conducir a una apariencia de edad facial avanzada.

3. Cantidad de hueso disminuida en la sínfisis mandibular.

4. Pacientes con problemas periodontales.

5. Reabsorción radicular, y

6. Relaciones esqueléticas de clase II y clase III severas.

\section{Consideraciones Generales para realizar una máxima retracción con mini-implantes}

Para realizar una máxima retracción con miniimplantes es necesario tener algunas consideraciones: $(5-7)$

a. Magnitud de apiñamiento, la extracción de dientes se realiza para aliviar apiñamientos severos y moderados, que comprometen la función y la estética facial del paciente; además, para camuflar problemas esqueléticos de clases II o III leves y moderados (5-7).

b. Vestibularización excesiva de los dientes anteriores, esto implica una necesidad de espacio adicional, independiente de la que se cuantifica en el apiñamiento y determina el tipo de movimiento en masa, que se debe hacer durante la fase de cierre. Movimiento que puede ser de inclinación y después de traslación. c. Discrepancias de la línea media, esto implica la necesidad de espacio hacia el lado contrario de la desviación. Se debe corregir antes de empezar el cierre de espacios.

d. Dimensión vertical, es importante poner atención a las fuerzas verticales resultantes de mecánicas anteroposteriores en el cierre de espacios en masa.

Las piezas posteriores se extruyen y aumentan la altura facial antero inferior e incrementan el espacio interlabial, y las anteriores aumentan la franja de sonrisa gingival.

\section{Consideraciones biomecánicas para realizar una máxima retracción con mini-implantes}

Algunas consideraciones biomecánicas deben tenerse en cuenta, estas son:

a. Cantidad de retracción de los dientes anteriores.Eluso de mini-implantes permite grandes retracciones, pero es necesario tomar en cuenta lo siguiente: la estética de paciente, cantidad de hueso disponible, estado de los tejidos periodontales, la longitud y anatomía radicular $(5,6)$.

b. Vectores de fuerzas de retracción y control vertical de los incisivos. - Las mecánicas de cierre del espacio tienden a aumentar la sobremordida, por tal motivo se recomienda añadir "ganchos" de retracción para controlar este efecto secundario (5). Los miniimplantes son instalados generalmente a $8 \mathrm{~mm}$ por encima de la unión cemento adamantina (UCA) hacia mesial de los primeros molares. Se debe considerar que al realizar una retracción anterior con anclaje directo al mini-implante, se genera un vector de fuerza más intrusivo que las mecánicas convencionales (5). Este vector de fuerza puede ser controlado mediante la modificación de la altura de instalación del miniimplante y/o la altura del "hook" generando una serie de posibilidades en las líneas de acción de la fuerza. Por lo tanto, el ortodoncista antes de instalar o indicar el uso de mini-implantes, debe definir la línea de acción que va a utilizar y el efecto vertical que esta mecánica tendrá (5).

Los vectores de fuerza pueden ser: a) máxima retracción con vector de fuerza bajo, b) máxima retracción con vector de fuerza intermedio, y c) 
máxima retracción con vector de fuerza alto (5)

A continuación se explicara cada uno de estos vectores:

a) Máxima retracción con vector de fuerza bajo.- $\mathrm{Se}$ obtiene generalmente, con la instalación del miniimplante a $8 \mathrm{~mm}$ de UCA y un gancho corto de $4 \mathrm{~mm}$ de altura en el segmento anterior. Este tipo de vector de fuerza tiende a causar un giro en el plano oclusal en sentido horario, ya que pasa por debajo del centro de rotación del sistema, tal como se ve en la imagen $(7,8)$ (Figura 1).

b) Máxima retracción con vector de fuerza intermedio.- Se utiliza en personas que presentan una sobremordida adecuada, cuando se desea poca o ninguna alteración del plano oclusal $(7,8)$. Para realizar este tipo de retracción se requiere colocar el mini-implante lo más apical posible y modificar la altura del gancho (8 $\mathrm{mm}$ aproximadamente) para redirigir el vector de la fuerza de retracción, de forma que este pase a través del centro de rotación del segmento anterior. Éste se encuentra entre las raíces del incisivo lateral y canino, a $6.76 \mathrm{~mm}$ por encima de la zona cervical, o a nivel del ápice radicular (7-13) (Figura 2).

c) Máxima retracción con vector de fuerza alto.Se obtiene, generalmente con el uso de un gancho largo en el segmento anterior, Melsen y Verna (7) recomendaron que la longitud del gancho sea de 10 $\mathrm{mm}$ desde el arco principal, pero existen diversas limitaciones anatómicas las cuales hacen que esto no sea clínicamente factible (13). Este tipo de vector de fuerza tiende a causar un giro en el plano oclusal en

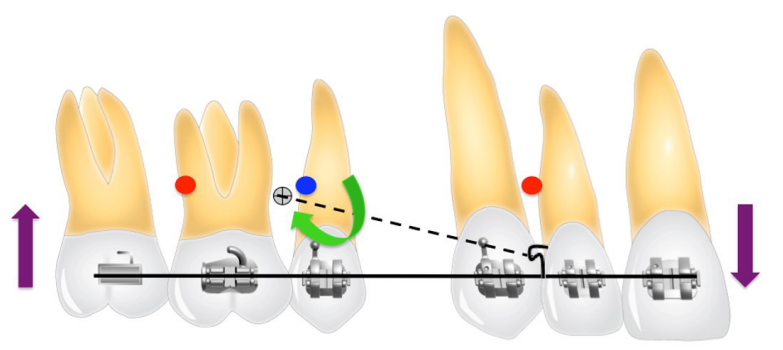

Figura 1. Modelo de máxima retracción anterosuperior (vector de fuerza bajo), se observa que la línea de acción de fuerza pasa por debajo del centro de rotación del sistema, provocando un giro en sentido horario. ${ }^{*}$ Punto rojo: centro de rotación de sector anterior y posterior respectivamente, ${ }^{*}$ Punto azul: centro de rotación de arcada completa. sentido anti-horario, ya que éste pasa por encima del centro de rotación del sistema (8) como se observa en la Figura 3.

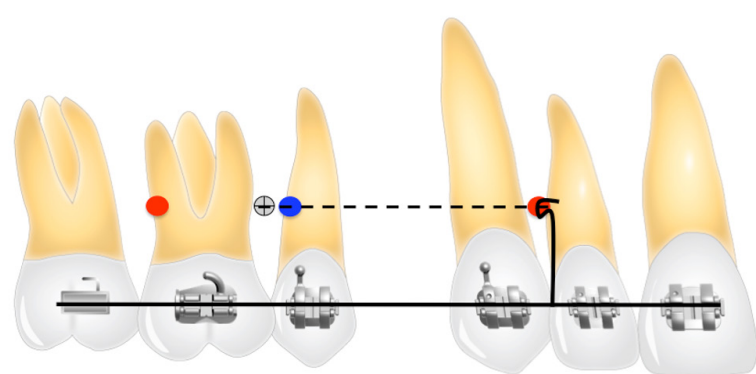

Figura 2. Modelo de máxima retracción anterosuperior (vector de fuerza intermedio), se observa que la línea de acción de fuerza pasa por el centro de rotación del sistema, provocando un movimiento de cuerpo entero. ${ }^{*}$ Punto rojo: centro de rotación de sector anterior y posterior respectivamente, ${ }^{*}$ Punto azul: centro de rotación de arcada completa.

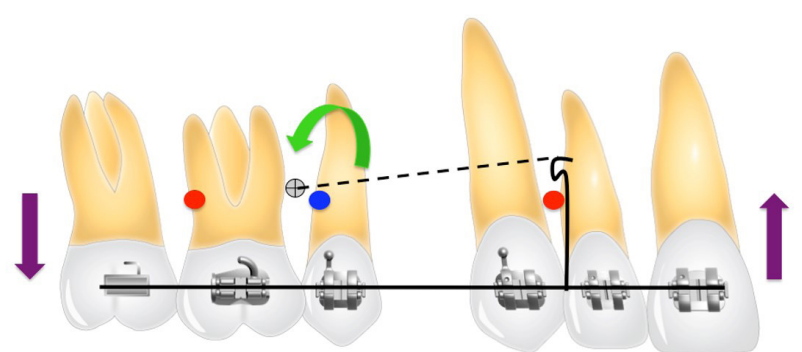

Figura 3. Modelo de máxima retracción anterosuperior (vector de fuerza alto), se observa que la línea de acción de fuerza pasa por encima del centro de rotación del sistema, provocando un giro del plano oclusal en sentido anti-horario. * Punto rojo: centro de rotación de sector anterior y posterior respectivamente, ${ }^{*}$ Punto azul: centro de rotación de arcada completa.

\section{REPORTE DE CASO}

Paciente femenino de 22 años de edad, acude al Servicio de Ortodoncia del Programa de Postgrado de la Facultad de Estomatología Roberto Beltrán, de la Universidad Peruana Cayetano Heredia, el motivo de consulta fue: "mis dientes de adelante están chuecos y salidos".

\section{Examen clínico}

Al examen clínico extraoral se observa una paciente dolicocéfala, dolicofacial, simétrica, tercio facial inferior aumentado, eversión del labio inferior, surco mentolabial marcado, perfil anteroposterior convexo, perfil vertical normodivergente, mentón 
poco prominente. Al examen intraoral se observa: relaciones molares clase II, relaciones caninas clase II, OJ de $5 \mathrm{~mm}$ y OB de $40 \%$. Ambos arcos son ovalados. La línea media superior centrada y la inferior desviada $3 \mathrm{~mm}$ a la derecha; se evidencia la presencia de un diente supernumerario (DSN) a nivel cervical de las piezas 16 y 17 (Figura 4).

\section{Exámenes auxiliares}

En la radiografía panorámica se observan estructuras y trabeculado óseo aparentemente normales; se aprecia dilaceración radicular de piezas 38 y 48 , piezas 46 y 47 mesioanguladas, imagen radiopaca (IRO) a nivel coronal de las piezas 16

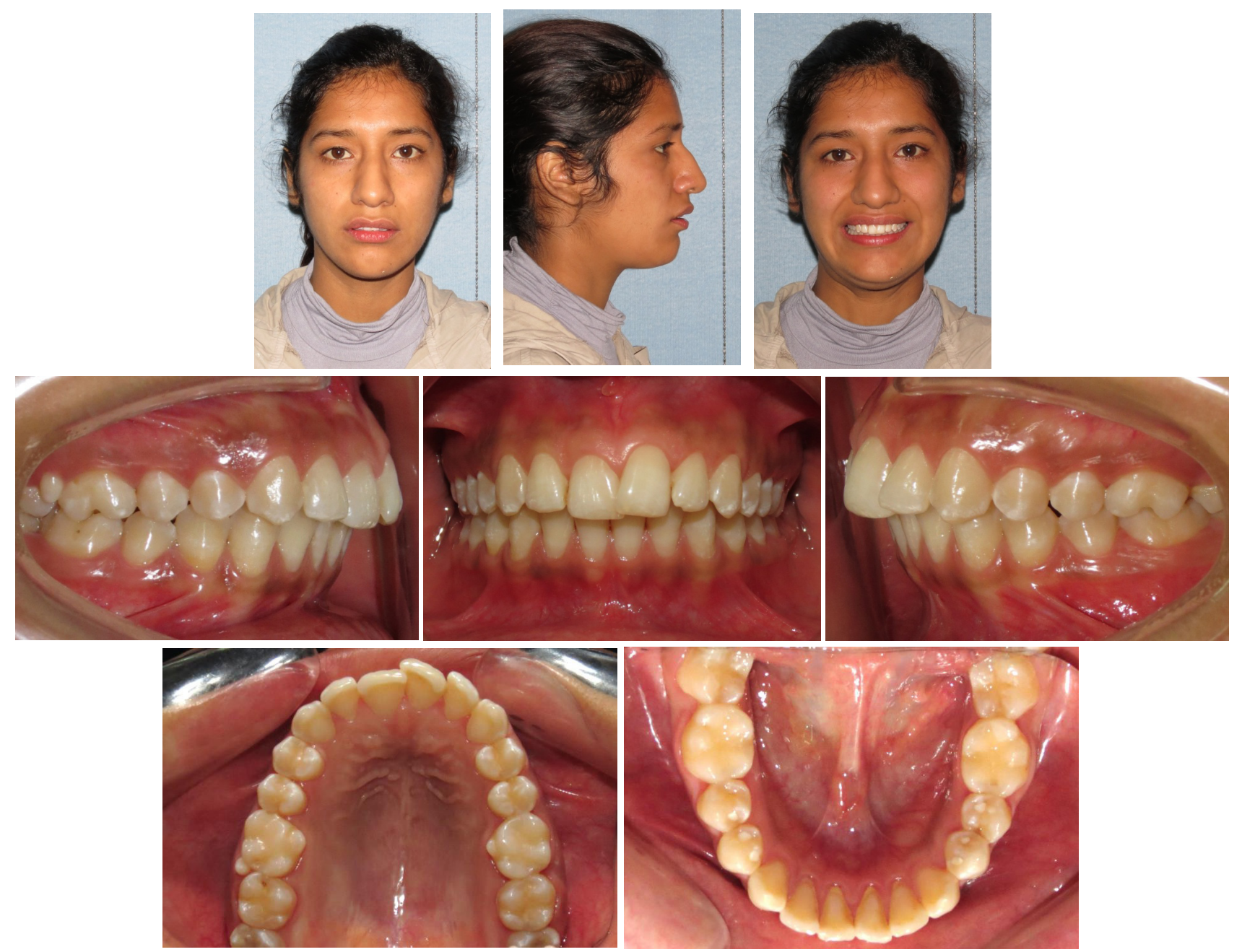

Figura 4. Set fotográfico inicial donde se observa: características extraorales e intraorales de la paciente.
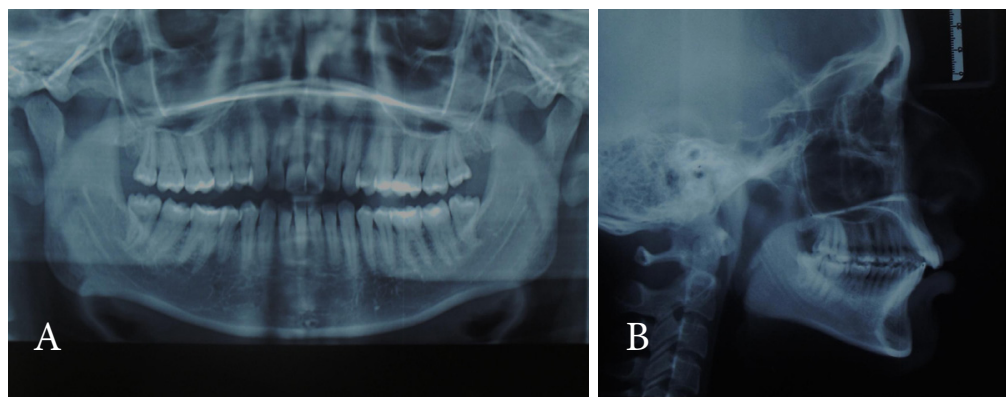

Figura 5. Radiografía pretratamiento. A. Radiografía panorámica, se observa dilaceración radicular de piezas: 38 y 48; piezas: 47 y 46 mesioanguladas. B. Radiografía cefalométrica lateral, se observa una relación esquelética clase II por protrusión maxilar. 
y 17 compatible con DSN. Con la radiografía cefalométrica se determina una relación esquelética clase II por protrusión maxilar, tendencia de crecimiento normodivergente y una altura facial anteroinferior aumentada, incisivos superiores e inferiores vestibularizados y protruidos (Figura 5). El análisis de modelos indica una discrepancia alveolo dentaria (DAD) superior de $-3 \mathrm{~mm}$ y una DAD inferior de $-1 \mathrm{~mm}$.

\section{Plan de tratamiento}

El tratamiento de este caso se programa con la extracción de las primeras premolares superiores y el uso de mini-implantes para obtener un anclaje máximo y realizar una máxima retracción con un vector de fuerza intermedio. Se utilizaron brackets metálicos preajustados de prescripción Roth con slot de $0.022 " x$ x 0.028 ".

\section{Evolución}

Luego de haber completado la fase de alineado y nivelado, se procedió a realizar las extracciones de las primeras premolares superiores y la colocación de los mini-implantes. Debido a las características oclusales de la paciente se realizó una máxima retracción con un vector de fuerza intermedio (explicado anteriormente).

Luego de haber realizado el cierre de espacios aplicando una fuerza de 250 gr. por lado con un arco 0.019 " x 0.025 " pulgadas de acero con pines soldados entre el incisivo lateral y canino, procedimos a tomar una radiografía lateral y panorámica para verificar el paralelismo radicular (Figuras 6 y 7, Tabla 1).

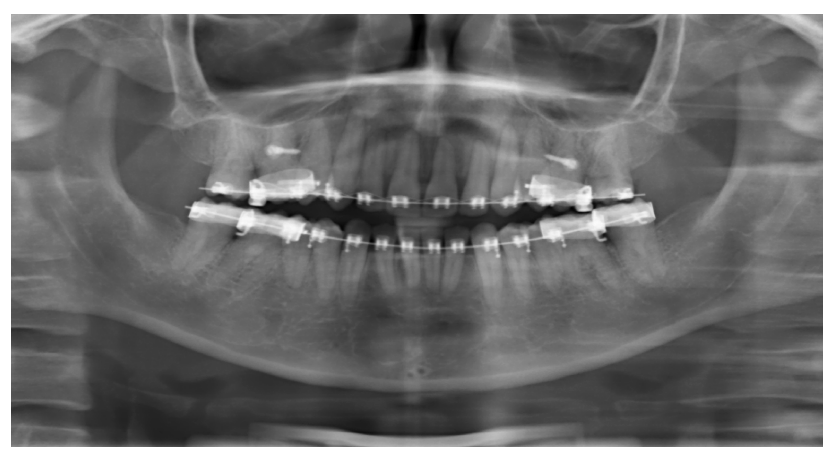

Figura 6. Radiografía panorámica de control, se observa el paralelismo radicular después de la mecánica de cierre de espacios.

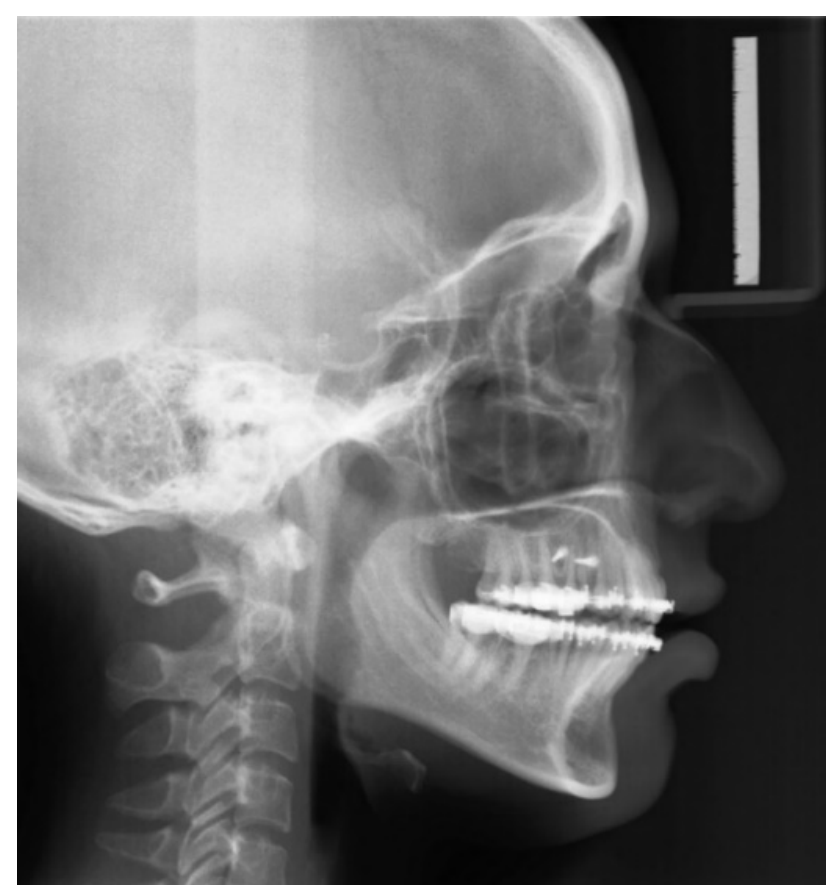

Figura 7. Radiografía cefalométrica lateral de control, se observa el cambio de angulación de piezas anterosuperiores.

Con la ayuda de la radiografía panorámica se realizaron algunas reposiciones de brackets para obtener el paralelismo radicular final y lograr una adecuada estabilidad oclusal.

De esta manera se inició la fase de acabado e intercuspidacion con un arco de acero de 0.019 " $\mathrm{x}$ 0.025 " y posteriormente se utilizó un arco de acero multi hebras Braided 0.019" x 0.025", asociados a elásticos intermaxilares (EIM) de $1 / 8$ utilizados en los sectores posteriores bilateralmente. Finalmente, se realizó la superposición de los trazados cefalométricos pretratamiento (trazado negro) y postratamiento

Tabla 1. Angulaciones pretratamiento y durante el tratamiento.

\begin{tabular}{ccc}
\hline Ángulo & $\begin{array}{c}\text { Antes de } \\
\text { tratamiento }\end{array}$ & $\begin{array}{c}\text { Durante } \\
\text { tratamiento }\end{array}$ \\
\hline I.na & $29^{\circ}$ & $12^{\circ}$ \\
I-na & $5^{\circ}$ & 0 \\
I.pp & $117^{\circ}$ & $100^{\circ}$ \\
Impa & $105^{\circ}$ & $104^{\circ}$ \\
I.i & $112^{\circ}$ & $126^{\circ}$ \\
Sna & $79^{\circ}$ & $79^{\circ}$ \\
Snb & $72^{\circ}$ & $72^{\circ}$ \\
\hline
\end{tabular}


(trazado rojo) para evaluar los cambios obtenidos (Figuras 8,9,10,11, Tabla 2).

\section{DISCUSIÓN}

El uso de mini-implantes para el refuerzo de anclaje ortodóncico se ha vuelto cada vez más popular en los últimos años, especialmente en adultos que no desean llevar aparatos extraorales. Los mini-implantes ahorran tiempo a través de un cierre de espacio en masa, y producen buenos resultados de tratamiento sin necesidad de la cooperación del paciente. Sin embargo, en algunos pacientes tratados con miniimplantes, los factores mecánicos pueden producir
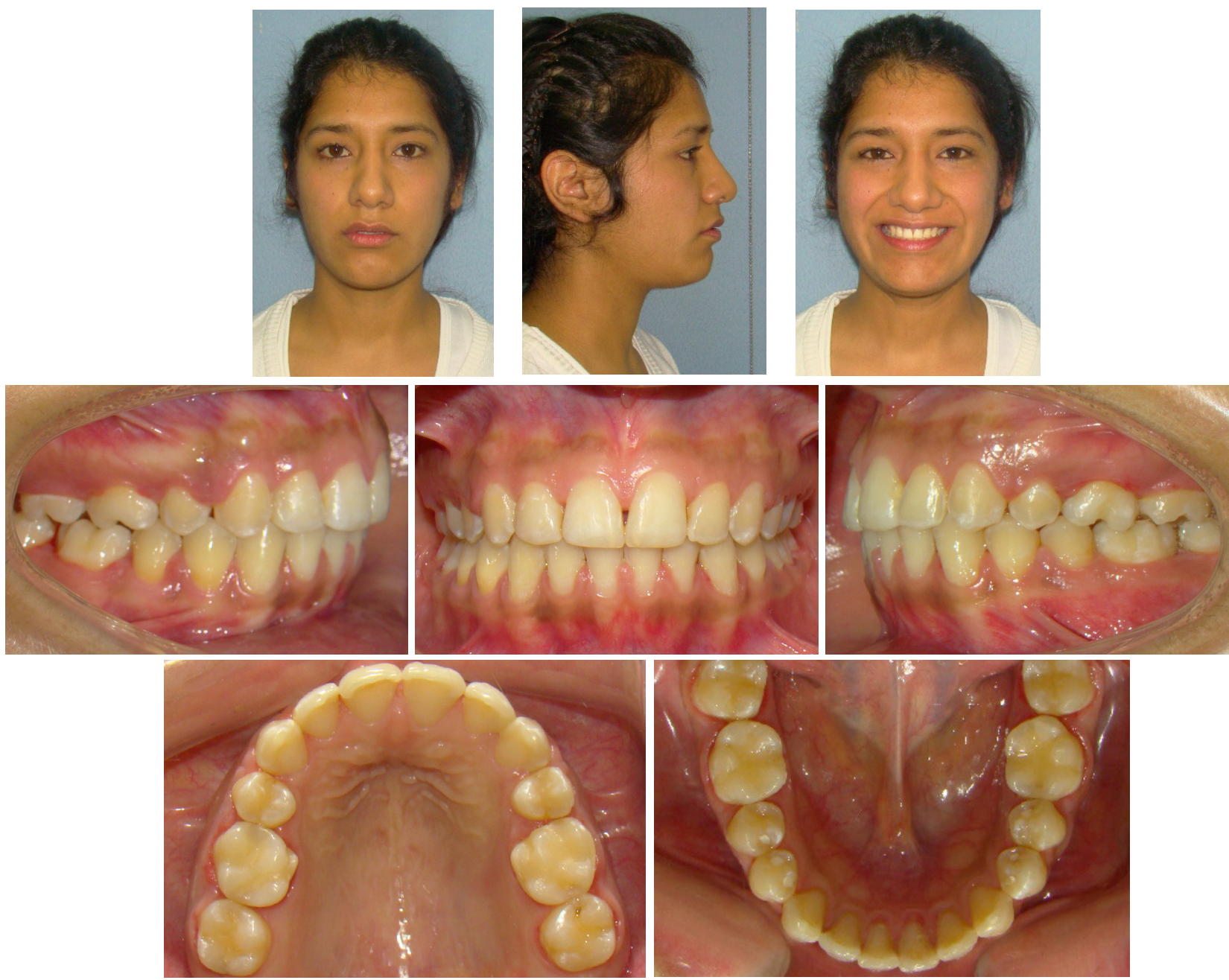

Figura 8. Set fotográfico donde se observan características extraorales e intraorales finales de paciente.

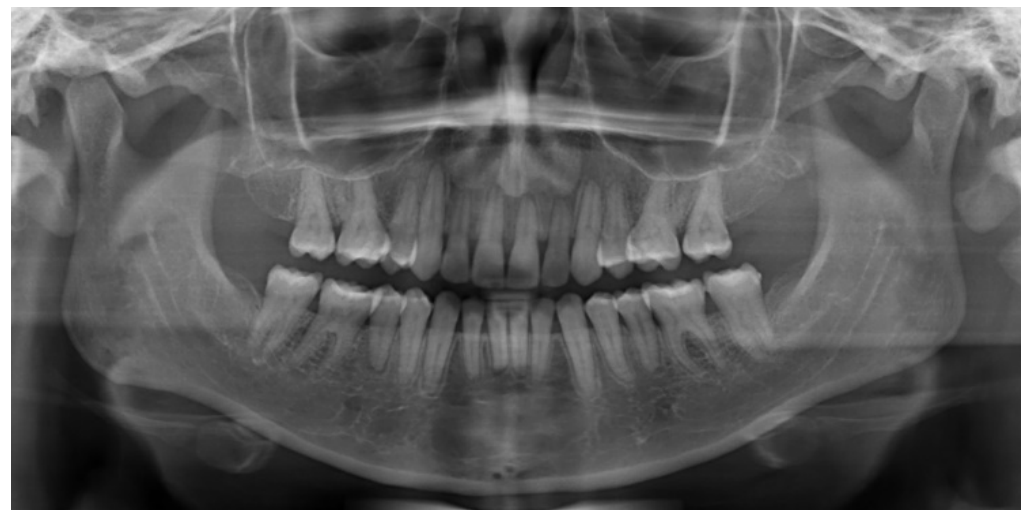

Figura 9. Radiografía panorámica postratamiento, se observa el paralelismo radicular post retiro de bracktes. Se realizó la extracción de piezas: 18,28,38,48. 


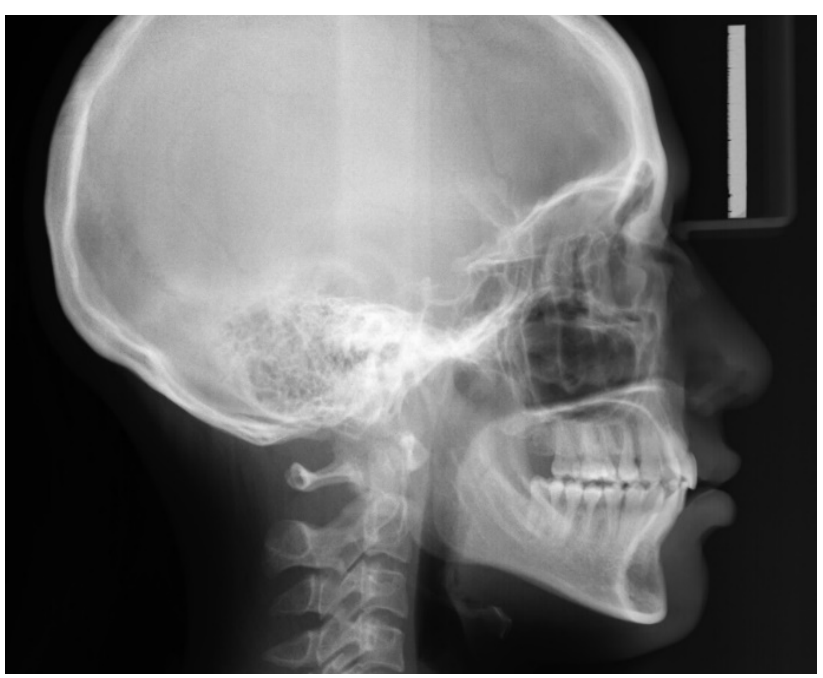

Figura 10. Radiografía cefalométrica lateral postratamiento, se observa cambio en angulación de piezas antero-superiores, las cuales se muestran en Tabla 2.

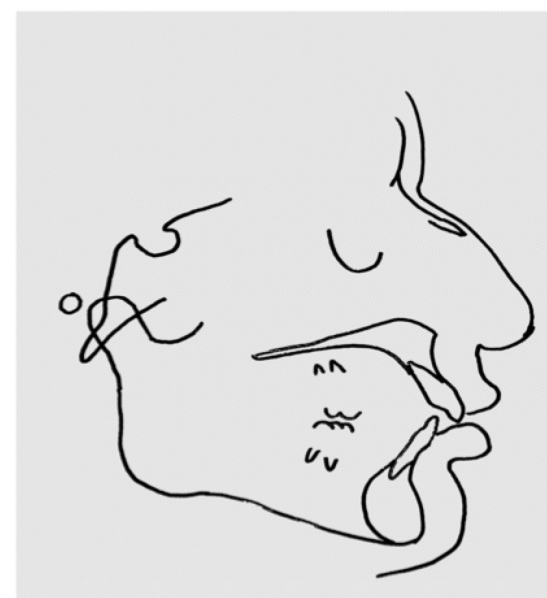

A

Figura 11. Trazado cefalométrico donde se observan los cambios obtenidos en angulaciones de incisivos. A: Inicial. B: Final.

Tabla 2. Angulaciones pretratamiento, durante el tratamiento y postratamiento.

\begin{tabular}{cccc}
\hline Ángulo & Antes de tratamiento & Durante tratamiento & Final de tratamiento \\
\hline I.NA & $29^{\circ}$ & $12^{\circ}$ & $21^{\circ}$ \\
I-NA & $5^{\circ}$ & 0 & $1^{\circ}$ \\
I.Pp & $117^{\circ}$ & $100^{\circ}$ & $110^{\circ}$ \\
IMPA & $105^{\circ}$ & $104^{\circ}$ & $105^{\circ}$ \\
I.I & $112^{\circ}$ & $126^{\circ}$ & $118^{\circ}$ \\
SNA & $79^{\circ}$ & $79^{\circ}$ & $79^{\circ}$ \\
SNB & $72^{\circ}$ & $72^{\circ}$ & $72^{\circ}$ \\
\hline
\end{tabular}


cambios o efectos secundarios inusuales, en el plano sagital, horizontal y transversal (14-16).

La retracción anterior con mecánica de deslizamiento convencional, generalmente se logra mediante la colocación de una cadena elastomérica o resortes de níquel desde el gancho del tubo de la primera molar al gancho soldado entre el incisivo lateral y el canino. En este caso, el segmento anterior y segmentos posteriores giran alrededor del centro de rotación, en sentido horario, lo que provoca una inclinación horaria del arco maxilar. El uso de mini-implantes como refuerzo de anclaje produce una modificación en la mecánica convencional, logramos que la línea de acción de fuerzas pase a través del centro de rotación minimizando efectos secundarios verticales (extrusión o intrusión del segmento anterior), obteniendo así un paralelismo entre el plano oclusal y la línea de acción de fuerzas (14-16).

\section{CONCLUSIONES}

La utilización de los mini-implantes, brinda un adecuado anclaje esquelético para realizar una máxima tracción durante la mecánica de cierre de espacios en masa por deslizamiento. Es importante conocer la influencia de las fuerzas aplicadas sobre el sistema para diseñar una correcta biomecánica y controlar los posibles efectos colaterales.

\section{Correspondencia}

Aylin Gallegos Salazar

Calle $54 \mathrm{~N}^{\circ} 173$, San Isidro. Lima, Perú.

Correo electrónico: aylingallegossalazar@gmail.com

\section{REFERENCIAS BIBLIOGRÁFICAS}

1. Nanda R, Kuhlberg A. Biomecánicas y estética, estrategias en ortodoncia clínica 1era ed. Caracas: Amolca; 2007.

2. Park HS, Kwon TG. Sliding mechanics with microscrew implant anchorage. Angle Orthod. 2004; 74(5):703-10.

3. Block MS, Hoffman DR. A new device for absolute anchorage for orthodontics. Am J Orthod Dentofacial Orthop. 1995; 107(3):251-8.
4. Uribe G. Ortodoncia Teoría y Clínica. 1ra ed. Medellin: CIB; 2004.

5. Marassi C. Mini-implantes ortodônticos como auxiliares da fase de retração anterior. R Dental Press Ortodon Ortop Facial. 2008; 13(5):57-75.

6. Marassi C. Carlo Marassi responde (parte I): Quais as principais aplicações clínicas e quais as chaves para o sucesso no uso de mini-implantes em Ortodontia? R Dental Press Ortodon Ortop Facial. 2006; 5(4):13-25.

7. Melsen B, Verna C. Miniscrew implants: the archus anchorage system. Semin Orthod. 2005; 11(1):24-31.

8. Carano A. Clinical applications of the miniscrew anchorage system. J Clin Orthod. 2005; 39(1):9-24.

9. Jung MH, Kim TW. Biomechanical considerations in treatment with miniscrew anchorage. Part 1: the sagittal plane. J Clin Orthod. 2008; 42(2):79-83.

10. Upadhyay M, Yadav S, Nagaraj K, Patil S. Treatment effects of mini-implants for en-masse retraction of anterior teeth in bialveolar dental protrusion patients: a randomized controlled trial. Am J Orthod Dentofacial Orthop. 2008; 134(1):18-29.

11. Papadopoulos MA, Christou PK. Centers of resistance of the maxillary complex: Theoretical models and practical applications in orthodontics. Hell Orthod Rev. 2000; 3(1):35-51.

12. Tominaga JY, Tanaka M, Koga Y, Gonzales C, Kobayashi M, Yoshida N. Optimal loading conditions for controlled movement of anterior teeth in sliding mechanics. Angle Orthod. 2009; 79(6):1102-7.

13. Lee HK, Chung KR. The vertical location of the center of resistance for maxillary six anterior teeth during retraction using three dimensional finite element analysis. Korean J Orthod. 2001; 31(1):425-38.

14. Kim TK, Kim JT, Mah J, Yang WS, Baek SH. First or second premolar extraction effects on facial vertical dimension. Angle Orthod. 2005; 75(2):177-82.

15. Ong HB, Woods MG. An occlusal and cephalometric analysis of maxillary first and second premolar extraction effects. Angle Orthod. 2001; 71(2):90-102.

16. Kocadereli I. The effect of first premolar extraction on vertical dimension. Am J Orthod Dentofacial Orthop. 1999; 116(1):41-5.

Recibido: 06/01/2015

Aceptado: 25/02/2015 